\title{
Toxic Leukoencephalopathy in a Teenager Caused by the Recreational Ingestion of 25I-NBOMe: A Case Report and Review of Literature
}

\author{
Chris Humston ${ }^{\mathrm{a}, \mathrm{c}}$, Renata Miketic ${ }^{\mathrm{a}, \mathrm{b}}$, Kelly Moon ${ }^{\mathrm{a}}$, Peter Ma ${ }^{\mathrm{a}, \mathrm{b}}$, \\ Joseph Tobias ${ }^{\mathrm{a}, \mathrm{b}}$
}

\begin{abstract}
Over the past 5 years, the designer drug classification of illicit substances has gained the attention of law enforcement agencies, healthcare providers, and concerned parents around the world. These drugs are often marketed as safe and legal alternatives to their already banned counterparts and are easily acquired online, at "head" shops or behind the counter at local convenient stores. This new drug class includes synthetic cathinones, synthetic cannabinoids, and phenylethylamine derivatives of the $2 \mathrm{C}$ class of hallucinogens. Many of these drugs were created for animal research purposes only and were never intended for human consumption. As such, little is known about their pharmacokinetic, pharmacodynamics, and adverse effect profile. Variability in preparation may further alter the already unpredictable safety profile. We report an otherwise healthy 16-year-old adolescent who presented to the emergency department for worsening left-sided weakness, new onset seizure activity, changes in mental status, and an overall decline in health. He self-reported the recreational ingestion of 25I-NBOMe prior to admission. Magnetic resonance imaging and a brain biopsy performed during the course of his admission confirmed the diagnosis of toxic leukoencephalopathy secondary to synthetic hallucinogenic drug use. The basic pharmacology and end-organ effects of 25I-NBOMe are reviewed, their adverse effect profile is presented, and potential anesthetic implications are postulated.
\end{abstract}

Keywords: 25I-NBOMe; N-Bomb; Designer drugs; Synthetic hallucinogen; Brain damage; Illicit drugs; Controlled substances; Toxic leukoencephalopathy

\footnotetext{
Manuscript accepted for publication April 18, 2017

aDepartment of Anesthesiology \& Pain Medicine, Nationwide Children's Hospital, Columbus, $\mathrm{OH}$, USA

${ }^{b}$ Department of Anesthesiology \& Pain Medicine, The Ohio State University College of Medicine, Columbus, OH, USA

${ }^{\mathrm{c}}$ Corresponding Author: Chris Humston, Department of Anesthesiology \& Pain Medicine, Nationwide Children's Hospital, 700 Children's Drive, Columbus, $\mathrm{OH} 43205$, USA.

Email: Christopher.Humston@nationwidechildrens.org
}

doi: https://doi.org/10.14740/jmc2811w

\section{Introduction}

Over the past 5 years, the designer drug class of illicit substances has gained the attention of law enforcement agencies, health care providers, and concerned parents around the world, due in part to increased media coverage and also due to governmental steps taken to combat their proliferation [1]. These drugs are often marketed as safe and legal alternatives to their controlled counterparts and can easily be acquired through various legal channels such as convenient stores, gas stations, "head shops", and via the internet. This new designer drug class includes synthetic cathinones (bath salts), synthetic cannabinoids (potpourri, spice, K2) and psychedelics of the NBOMe series of substituted phenylethylamine derivatives $[2,3]$.

The NBOMe series of drugs are extremely potent hallucinogens originally created for research purposes and use in laboratory animals. They were never intended for human consumption. As such, their physiological and toxic effects have not been studied in humans resulting in an unpredictable and unknown safety profile. Inadvertent consumption has resulted in a recent spike in toxicology reports regarding individuals who mistakenly ingested 25I-NBOMe instead of LSD, resulting in death [2]. Although anecdotal case reports exist in toxicology and forensic journals regarding the dangers of 25INBOMe ingestion, we report for the first time, the need for anesthetic care of a patient who had ingested 25I-NBOMe. The basic pharmacology and end-organ effects of 25I-NBOMe are reviewed, their adverse effect profile is presented, and potential anesthetic implications are postulated.

\section{Case Report}

Institutional Review Board approval is not required by Nationwide Children's Hospital for presentation of single case reports. The patient, a 16-year-old, $54.2 \mathrm{~kg}$ adolescent, presented to the emergency department (ED) for assessment due to recent neurological changes and abnormal findings on magnetic resonance imaging (MRI) at an outside facility. Approximately, 2.5 weeks prior to this encounter, the patient was seen by his primary care physician (PCP) for complaints of left-sided weakness and was diagnosed with a conversion disorder. A few days after the visit with his PCP, he was brought to the ED 
for re-evaluation of his symptoms. He was evaluated by the Pediatric Neurology Service, who agreed with the diagnosis of conversion disorder and recommended follow-up with Psychiatry. Over the next 2 weeks, his neurological changes and deficits that started with left-sided motor weakness progressed to include left-sided sensory changes, a facial droop, altered gate, and seizure-like activity. All of these changes had been intermittent yet progressive over the past 2 weeks. Per his parents, the patient had also been noted to have personality changes, which included being more defiant and argumentative. During the assessment in the ED, although the patient was alert and oriented, he had some difficulty following commands. His left arm and left leg were significantly weaker than the right side. No facial asymmetry was noted. He did not display aggressive behavior, nor was he an immediate threat to himself. His vital signs were stable and included a resting respiratory rate of 28 breaths/min, a room-air oxygen saturation of $98 \%$ by pulse oximetry, a heart rate of 88 beats $/ \mathrm{min}$, and a blood pressure of $143 / 83 \mathrm{~mm} \mathrm{Hg}$. Initial laboratory values, consisting of a general chemistry (sodium $140 \mathrm{mEq} / \mathrm{L}$, potassium $3.9 \mathrm{mEq} / \mathrm{L}$, chloride $104 \mathrm{mEq} / \mathrm{L}$, carbon dioxide 23 $\mathrm{mEq} / \mathrm{L}, \mathrm{BUN} 9 \mathrm{mg} / \mathrm{dL}$, and creatinine $0.68 \mathrm{mg} / \mathrm{dL}$ ), a complete blood count, and a coagulation panel (prothrombin time, activated thromboplastin time, international normalized ration, and fibrinogen) were all within normal limits. His past medical history was significant for attention deficit hyperactivity disorder, depression, and a remote history of asthma as a child. No home medications were reported. His seizure history included a single episode of seizure activity (tonic-clonic activity) after the recreational ingestion of 25I-NBOMe approximately 18 months prior to this admission. Since that time, the patient reported intermittent hallucinations and seizure-like activity, but no medical attention had been sought for these complaints. Even after direct questioning, the patient did not provide additional information regarding the frequency with which he used 25I-NBOMe or when the last time was that he had used it. Given the history of ongoing problems and the findings on physical examination in the ED, the decision was made to obtain an urgent MRI examination. The patient demonstrated persistent shivering and shaking without definitive seizure activity. He also would involuntarily rotate his head clockwise, as if doing neck exercises. For this reason, the radiology and neurology team requested general anesthesia in order to obtain optimum MRI quality. During the preoperative physical examination, the patient was noted to be nonverbal, but nodded appropriately to questions and appropriately followed commands. The remainder of the physical examination was unremarkable with a normal airway, respiratory and cardiovascular examination. His vital signs were unremarkable. When moving from his hospital bed to the MRI scanner table, he developed severe ataxia, requiring assistance. In addition, his left foot exhibited clonus, appearing to be permanently plantar flexed, making it difficult to stand or walk. Due to the patient's clinical presentation and unknown NPO status, a rapid sequence induction (RSI) was performed after the administration of intravenous lidocaine (50 $\mathrm{mg})$, propofol $(150 \mathrm{mg})$, and rocuronium $(50 \mathrm{mg})$. Endotracheal intubation was easily achieved using a Macintosh 3 blade and a cuffed styleted $7.0 \mathrm{~mm}$ endotracheal tube, with

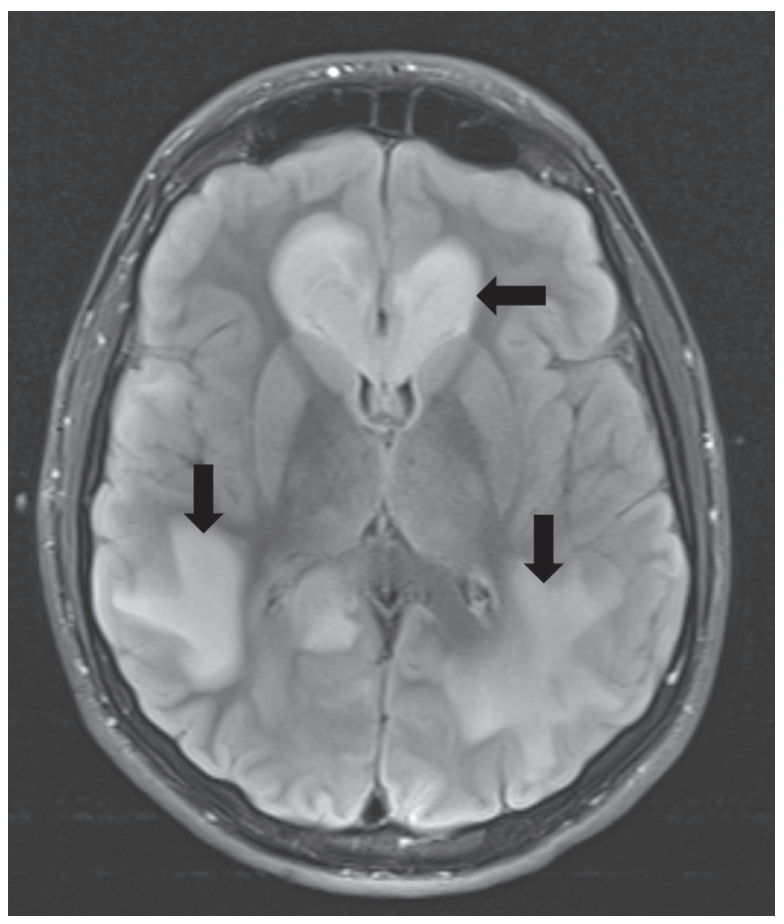

Figure 1. Magnetic resonance imaging of the brain with increased T2weighted density (black arrows) demonstrating toxic leukoencephalopathy involving the cerebral hemispheres.

a Cormack-Lehane score of I. Cricoid pressure was applied during induction and was released upon confirmation of bilateral breath sounds and a positive end-tidal carbon dioxide $\left(\mathrm{ETCO}_{2}\right)$ tracing. The endotracheal tube was taped and secured at a depth of $21 \mathrm{~cm}$ at the teeth. The patient tolerated anesthetic induction and endotracheal intubation without adverse hemodynamic effects. Dexamethasone $(8 \mathrm{mg})$ was administered for prophylaxis of postoperative nausea and vomiting. Mechanical ventilation was initiated for the case and anesthesia was maintained with inhaled sevoflurane (exhaled concentration 2-3\%). With the exception of one episode of transient hypotension (BP 75/50) approximately 45 min after induction of anesthesia requiring the administration of phenylephrine $(100 \mu \mathrm{g})$, the anesthetic was uneventful. Upon completion of the imaging, ondansetron (4 mg) was administered for antiemetic prophylaxis. Residual neuromuscular blockade was reversed with glycopyrrolate $(0.4 \mathrm{mg})$ and neostigmine (3 mg). During emergence and prior to extubation of the trachea, the patient demonstrated seizure-like activity (tonic-clonic movements of his arms and legs) and urinary incontinence. Midazolam (2 mg) was administered which terminated the activity. Shortly after this, the patient's trachea was extubated when he was awake and following commands. The patient was transferred to the post-anesthesia care unit (PACU) breathing spontaneously on $100 \%$ oxygen via a face mask. The MRI revealed large zones of signal abnormality involving both cerebral hemispheres, suggestive of acute versus subacute leukoencephalopathy (Fig. 1). Despite these findings, the exact etiology was not readily apparent. The subsequent workup did not provide any additional information as 
to the etiology of the patient's disease process. Tests results for West Nile virus, mercury levels, human immunodeficiency virus (HIV), and arsenic were all negative. Assays were also negative for synthetic cannabinoid metabolites, bath salts, and NMDA receptor antibodies. Cerebrospinal fluid analysis was negative for bacteria, mycoplasma, herpes simplex virus (HSV), and cytomegalovirus (CMV). Blood serology including an encephalopathy panel was also negative. Based on the lack of an etiology, the neurosurgery and neurology consultants determined that a brain biopsy would be needed to provide a definitive diagnosis. The patient underwent a second general anesthetic the following week. Due to the patient experiencing recent nausea and vomiting, an RSI was performed. Anesthesia was induced with midazolam $(2 \mathrm{mg})$, fentanyl $(100 \mu \mathrm{g})$, and propofol $(200 \mathrm{mg})$ and neuromuscular blockade provided by succinylcholine $(120 \mathrm{mg}$ ). Endotracheal intubation was again easily achieved using a Macintosh 3 blade and a cuffed, styletted $7.0 \mathrm{~mm}$ endotracheal tube, with a Cormack-Lehane score of I. Cricoid pressure was applied during induction and was released upon confirmation of bilateral breath sounds and $\mathrm{ETCO}_{2}$ tracing. The endotracheal tube was taped and secured at a depth of $21 \mathrm{~cm}$ at the teeth. The patient tolerated anesthetic induction and endotracheal intubation without adverse hemodynamic effects. Anesthesia was maintained with desflurane $3 \%$ and a remifentanil infusion to ensure a rapid emergence for neurologic assessment upon completion of surgery. Intermittent doses of rocuronium were administered to maintain 1 twitch of the train-of-four. Per the request of the neurosurgeon, dexamethasone $(8 \mathrm{mg})$ and mannitol were administered prior to opening of the dura. Overall, the intraoperative anesthetic course was uneventful. At the completion of the case, remifentanil and desflurane were titrated down and then decreased. Hydromorphone (total dose $=0.5 \mathrm{mg}$ ) was titrated to respiratory rate for postoperative analgesia. Residual neuromuscular blockade was reversed with sugammadex. The patient emerged quickly from anesthesia and his trachea was extubated upon awakening. He was transported to PACU where he returned to his baseline neurologic status. His PACU stay was uneventful and he was transported to the inpatient ward.

The biopsies were taken from a frontal lobe lesion and showed non-specific hypercelluarity with some reaction changes and occasional atypical nuclei. There was no evidence of infection or granulomatous tissue. There were multiple zones of demyelination identified. The histologic findings were non-specific and based on the lack of infectious etiology to explain the findings, the provisional diagnosis is toxic leuekoencephalopathy from the ingestion of 25I-NBOMe. Three months after the 2-month hospitalization, the patient continued to receive extensive medical therapy including visits with Neurology, Psychiatry, Physical Medicine and Rehabilitation, Occupational Therapy and Physical Therapy. He was still experiencing seizure activity and was seen in the ED 2 weeks after his discharge for a generalized tonic-clonic seizure which was treated with intranasal midazolam. To prevent further seizure activity, oxcarbazepine was started, but compliance had been intermittent due to adverse effects from the medication. Repeat MRI showed no acute changes from previous studies. He denied further illicit drug abuse. Physical examination showed ongoing left-sided weakness and a tremor in the left upper extremity. The patient had difficulty with activities of daily living such as tying his shoes and getting dressed, but he reported improvement with occupational and physical therapy. $\mathrm{He}$ also continued to display higher executive dysfunction such as problems with normal conversation. Multiple medical providers had not provided a definitive prognostic outlook.

\section{Discussion}

Originally synthesized in 2003 by Ralph Heim at the Free University of Berlin and further developed by David Nichols at Purdue University, the NBOMe series of synthetic hallucinogens were initially developed during the study of the serotonin receptors and their role on normal cognition [4]. A radiolabeled version of 25I-NBOMe has been validated for use as a PET scan radiotracer in various neurological imaging procedures [5]. NBOMe is a full agonist of the serotonin receptor subtype, $5-\mathrm{HT}_{2 \mathrm{~A}}$ while classic psychedelics are only considered partial agonists $[1,6,7]$. This receptor is responsible for complex behaviors and thought processes, from working memory to affective disorders such as depression and schizophrenia [7]. Stimulation of this receptor produces the hallucinogenic effects recreational users are seeking, such as euphoria, mystical experiences, vivid open and closed eye visuals, alterations in cognition, and powerful sensory and somatic effects $[1,7]$.

The NBOMe family of synthetic hallucinogens is derived from the $2 \mathrm{C}$ class of phenethylamines, including NMDA (Ectasy) and the newer 2C-I, 2C-B, and 2C-C [2, 6, 7]. By adding an $\mathrm{N}$-methoxybenzyl group to the $2 \mathrm{C}$ compound, $\mathrm{NBOMe}$ is synthesized. The addition of the N-methoxybenzyl group to the $2 \mathrm{C}$ compound significantly increases the compounds affinity for the 5- $\mathrm{HT}_{2 \mathrm{~A}}$ receptor. To illustrate the increase in potency from the parent compound to 25I-NBOMe, a normal clinical dose of $2 \mathrm{C}-\mathrm{I}$ is $20 \mathrm{mg}$, whereas the common dose of 25I-NBOMe is as little as $50-100 \mu \mathrm{g}$ [7-9]. Common routes of administration include the sublingual, buccal, and intranasal routes. Bioavailability is greater with intranasal administration compared to buccal or sublingual routes. Doses and concentrations often vary depending on the formulation of the drug, the product that contains it, and how it is applied to the mucous membranes (blotter paper, powder or crystal form). These variations in dosing and applications increase the likelihood of toxicity and fatal overdoses which are appearing anecdotally in the medical literature [8-10]. Although users may think that blotter paper laced with $25 \mathrm{I}-\mathrm{NBOMe}$ is the safest method of ingestion, since each square is thought to contain a pre-measured amount of the drug, manufacturer variability and lack of production standards result in significant variations in drug concentrations resulting in the reports of fatal overdoses from all forms and routes of administration of 25I-NBOMe.

NBOMe contains both stimulant and hallucinogenic properties and is a full agonist at the $5-\mathrm{HT}_{2 \mathrm{~A}}$ receptor site. The serotonin receptors, also known as 5-hydroxytryptamine receptors or 5-HT receptors, are a group of G protein-coupled receptors (GPCRs) and ligand-gated ion channels (LGICs) found 
Table 1. Signs and Symptoms of Serotonin Syndrome

\begin{tabular}{l} 
1. Agitation and akathisia \\
2. Repetitive head rotating with neck in moderate extension \\
3. Hyperreflexia and hypertonicity (greater in lower extremity) \\
4. Clonus (greater in lower extremity) \\
5. Myoclonus \\
6. Tremor (greater in lower extremity) \\
7. Hyperthermia \\
8. Shivering \\
9. Tachycardia \\
10. Mydriasis \\
11. Diaphoresis \\
12. Increased bowel sounds and diarrhea \\
\hline
\end{tabular}

in the central and peripheral nervous systems. There are seven 5-HT receptors found in the body that mediate both excitatory and inhibitory neurotransmission. Serotonin is responsible for many functions in the body including platelet aggregation, gastrointestinal motility, vasoconstriction, bronchoconstriction, mood, appetite, wakefulness, and personality [11-13]. Currently, 25I-NBOMe is considered one of the most potent $5-\mathrm{HT}_{2 \mathrm{~A}}$ receptor agonists in the world [8-10].

Patients with 25I-NBOMe toxicity frequently display signs of serotonin syndrome (Table 1) [11-14]. Serotonin syndrome is a potentially fatal complication resulting from the overstimulation of serotonin receptors in the body. The increase in serotonergic activity can lead to mental status changes, hypertonicity and hyperreflexia with clonus and rigidity, and autonomic hyperactivity. This may progress to life-threatening issues including multiple organ dysfunction, shock, metabolic acidosis, rhabdomyolysis, disseminated intravascular coagulation, renal failure, acute respiratory distress syndrome, and hyperthermia. $5-\mathrm{HT}_{1 \mathrm{~A}}$ and $5-\mathrm{HT}_{2 \mathrm{~A}}$ are the receptors thought to be most responsible for serotonin syndrome [15]. Serotonin syndrome may occur in patients of any age and severe systemic manifestations may be seen within minutes of ingestion of a causative agent $[13,15]$. Given its rapid progression, early and accurate diagnosis is essential.

Serotonin syndrome classically has been diagnosed on the basis of a triad of symptoms consisting of autonomic dysfunction, neuromuscular excitation, and altered mental status $[13,15]$. Recently, two tools have been established to help healthcare professionals ensure an accurate diagnosis of the syndrome, the Hunter and the Sternbach criteria [16-18]. Both

Table 2. The Hunter Criteria for Serotonin Syndrome

Ingestion of a known serotonergic agent and:

1. Spontaneous clonus

2. Inducible clonus or ocular clonus and agitation of diaphoresis

3. Inducible clonus or ocular clonus and hypertonicity and temperature $\geq 38^{\circ} \mathrm{C}$

4. Tremor and hyperreflexia
Table 3. Sternbach's Criteria

Ingestion of a known serotonergic agent and:

1. Absence of other etiologies

2. No recent addition or increase of a neuroleptic agent

3. The presence of three of the following signs and symptoms:
a. Mental status changes
b. Agitation
c. Myoclonus
d. Hyperreflexia
e. Diaphoresis
f. Shivering
g. Tremor
h. Diarrhea
i. Incoordination
j. Fever

include a constellation of symptoms that must be present with a known ingestion of a known serotonergic agent (Tables 2 and 3 ). The Hunter criterion is thought to be the preferred tool as it is less likely to produce false positives. The diagnosis of serotonin syndrome is purely clinical as laboratory results provide no diagnostic evidence. Serum serotonin levels have not been found to correlate with the findings of serotonin syndrome. Our patient exhibited many of the classic symptoms including spontaneous clonus, hyperreflexia, and tremors with ingestion of a known serotonergic agent. The presentation of serotonin syndrome mirrors many of the same signs and symptoms as other life-threatening conditions including malignant hyperthermia, anticholinergic syndrome, and neuroleptic malignant syndrome [15]. Differentiation between these medical issues is essential as treatment differs depending on the diagnosis. Serotonin syndrome may frequently be under recognized during anesthesia because the non-specific manifestations are attributed to the other causes and mild symptoms may be overlooked or attributed to other causes.

From 1999 to 2010, there has been a significant increase in the use of medications that act through or have effects on serotonin receptors and levels including a large list of anti-depressant medications including monoamine oxidase inhibitor (MAOI) or selective serotonin reuptake inhibitor (SSRI) [19]. This has led to an increased incidence of serotonin syndrome. Serotonin syndrome can occur when these medications are consumed in excess or taken in combination with other agents having serotonergic effects including illicit drugs. During the perioperative period, various medications may have serotonergic effects including opioids in the phenylpiperidine class (fentanyl, tramadol, meperidine, and methadone) and ondansetron. The phenylpiperidine class of opioids are not only agonists at the opioid receptor, but also weak serotonin reuptake inhibitors. Ondansetron may increase systemic serotonin levels via its blockade of $5-\mathrm{HT}_{3}$ receptors, leading to stimulation of other serotonin receptor subtypes [12]. Treatment of serotonin syndrome includes removal of the offending agent, 
avoidance or other medications with serotonergic effects, and treatment of the symptomatology. This may include control of agitation and hypertonicity with sedatives such as benzodiazepines, the use of vasoactive agents to control autonomic instability, maintenance of adequate diuresis to prevent the renal complications of rhabdomyolysis, and correction of hyperthermia [11]. As noted previously, early identification is essential. Prior to an accurate diagnosis, our patient received multiple pharmacologic agents that could have exacerbated the serotonin syndrome from 25I-NBOMe ingestion including ingested fentanyl and ondansetron. Avoidance of these medications may have been prudent with the concern of possible serotonin syndrome.

A unique finding in our patient was the finding of leukoencephalopathy on MRI. Leukoencephalopathy results from structural alterations of cerebral white matter, in which myelin is damaged [20]. There are many causative agents of toxic leukoencephalopathy, including environmental toxins (carbon monoxide, toluene), cranial irradiation, therapeutic agents (chemotherapy, amphotericin B), and drugs of abuse (cocaine, alcohol, heroin, ecstasy, cocaine and psilocybin). Clinical manifestations range from confusion, changes in personality, memory and attention deficits in milder cases to abulia, akinetic mutism, stupor, coma, severe global impairment and death in severe cases. The most distinct clinical manifestation remains mental status changes. MRI changes, as noted in our patient, consistent with the diagnosis of toxic leukoencephalopathy include diffuse and severe hyper-intensity of white matter, which may progress to focal areas of necrosis. Treatment options are limited, with the focus being on prevention or rehabilitation depending on the severity of the damage.

25I-NBOMe, also known as 25I, N-BOMB and Smiles is a designer synthetic hallucinogen which acts through the serotonin receptor, $5-\mathrm{HT}_{2 \mathrm{~A}}$ [21]. Toxicity is primarily related to the exaggerated serotonergic effects with clinical effects including seizures, hypertonicity, hyperthermia, and rhabdomyolysis. Anecdotal experience in our patient also suggests that long term use may result in permanent and irreversible damage to the central nervous system manifested as white matter changes including leukoencephalopathy. Anesthesia providers should maintain a high level of suspicion for NBOME use in any patient presenting with serotonin syndrome-like manifestations while reporting the recent use of recreational drugs. As no antagonists currently exist, treatment for NBOMe intoxication remains largely supportive. Until effective governmental measures eradicate the designer drug class of illicit substances, health care providers will likely see an increase in the number of patients presenting with serious adverse effects related to their use.

\section{References}

1. Ninnemann A, Stuart GL. The NBOMe series: a novel, dangerous group of hallucinogenic drugs. J Stud Alcohol Drugs. 2013;74(6):977-978.

2. Rose SR, Poklis JL, Poklis A. A case of 25I-NBOMe (25I) intoxication: a new potent 5-HT2A agonist designer drug. Clin Toxicol (Phila). 2013;51(3):174-177.
3. Abbott R, Smith DE. The New Designer Drug Wave: A Clinical, Toxicological, and Legal Analysis. J Psychoactive Drugs. 2015;47(5):368-371.

4. Poklis JL, Devers KG, Arbefeville EF, Pearson JM, Houston E, Poklis A. Postmortem detection of 25I-NBOMe [2-(4-iodo-2,5-dimethoxyphenyl)-N-[(2-methoxyphenyl) methyl] ethanamine] in fluids and tissues determined by high performance liquid chromatography with tandem mass spectrometry from a traumatic death. Forensic Sci Int. 2014;234:e14-20.

5. Ettrup A, Palner M, Gillings N, Santini MA, Hansen M, Kornum BR, Rasmussen LK, et al. Radiosynthesis and evaluation of 11C-CIMBI-5 as a 5-HT2A receptor agonist radioligand for PET. J Nucl Med. 2010;51(11):17631770.

6. Suzuki J, Dekker MA, Valenti ES, Arbelo Cruz FA, Correa AM, Poklis JL, Poklis A. Toxicities associated with NBOMe ingestion-a novel class of potent hallucinogens: a review of the literature. Psychosomatics. 2015;56(2):129139.

7. Braden MR, Parrish JC, Naylor JC, Nichols DE. Molecular interaction of serotonin 5-HT2A receptor residues Phe339(6.51) and Phe340(6.52) with superpotent N-benzyl phenethylamine agonists. Mol Pharmacol. 2006;70(6):1956-1964.

8. Kueppers VB, Cooke CT. 25I-NBOMe related death in Australia: a case report. Forensic Sci Int. 2015;249:e1518.

9. Gee P, Schep LJ, Jensen BP, Moore G, Barrington S. Case series: toxicity from 25B-NBOMe - a cluster of N-bomb cases. Clin Toxicol (Phila). 2016;54(2):141-146.

10. Andreasen MF, Telving R, Rosendal I, Eg MB, Hasselstrom JB, Andersen LV. A fatal poisoning involving 25C-NBOMe. Forensic Sci Int. 2015;251:e1-8.

11. Boyer EW, Shannon M. The serotonin syndrome. N Engl J Med. 2005;352(11):1112-1120.

12. Gollapudy S, Kumar V, Dhamee MS. A case of serotonin syndrome precipitated by fentanyl and ondansetron in a patient receiving paroxetine, duloxetine, and bupropion. J Clin Anesth. 2012;24(3):251-252.

13. Wilson L, Rooney T, Baugh RF, Millington B. Recognition and management of perioperative serotonin syndrome. Am J Otolaryngol. 2012;33(3):319-321.

14. Nicolaou G, Lee D. Methylene blue-induced serotonin syndrome presenting with ocular clonus and failure of emergence from general anesthesia. Can J Anaesth. 2016;63(7):896-897.

15. Wang RZ, Vashistha V, Kaur S, Houchens NW. Serotonin syndrome: Preventing, recognizing, and treating it. Cleve Clin J Med. 2016;83(11):810-817.

16. Sternbach H. The serotonin syndrome. Am J Psychiatry. 1991;148(6):705-713.

17. Dunkley EJ, Isbister GK, Sibbritt D, Dawson AH, Whyte IM. The Hunter Serotonin Toxicity Criteria: simple and accurate diagnostic decision rules for serotonin toxicity. QJM. 2003;96(9):635-642.

18. Bodner RA, Lynch T, Lewis L, Kahn D. Serotonin syndrome. Neurology. 1995;45(2):219-223.

19. Mojtabai R, Olfson M. National trends in long-term use 
of antidepressant medications: results from the U.S. National Health and Nutrition Examination Survey. J Clin Psychiatry. 2014;75(2):169-177.

20. Filley CM, Kleinschmidt-DeMasters BK. Toxic leukoencephalopathy. N Engl J Med. 2001;345(6):425-432.
21. Lawn W, Barratt M, Williams M, Horne A, Winstock A. The NBOMe hallucinogenic drug series: Patterns of use, characteristics of users and self-reported effects in a large international sample. J Psychopharmacol. 2014;28(8):780-788. 